\title{
Surprises from Mathematics Education Research: Student (Mis)use of Mathematical Definitions
}

\author{
Barbara S. Edwards and Michael B. Ward
}

1. INTRODUCTION. The authors of this paper met at a summer institute sponsored by the Oregon Collaborative for Excellence in the Preparation of Teachers (OCEPT). Edwards is a researcher in undergraduate mathematics education. Ward, a pure mathematician teaching at an undergraduate institution, had had little exposure to mathematics education research prior to the OCEPT program. At the institute, Edwards described to Ward the results of her Ph.D. dissertation [5] on student understanding and use of definitions in undergraduate real analysis. In that study, tasks involving the definitions of "limit" and "continuity," for example, were problematic for some of the students. Ward's intuitive reaction was that those words were "loaded" with connotations from their nonmathematical use and from their less than completely rigorous use in elementary calculus. He said, "I'll bet students have less difficulty or, at least, different difficulties with definitions in abstract algebra. The words there, like 'group' and 'coset,' are not so loaded."

Eventually, with OCEPT support, the authors studied student understanding and use of definitions in an introductory abstract algebra course populated by undergraduate mathematics majors and taught by Ward. The "surprises" in the title are outcomes that surprised Ward, among others. He was surprised to see his algebra students having difficulties very similar to those of Edwards's analysis students. (So he lost his bet.) In particular, he was surprised to see difficulties arising from the students' understanding of the very nature of mathematical definitions, not just from the content of the definitions. Upon hearing of Edwards's dissertation work, some other mathematicians who teach undergraduates found those difficulties surprising even when restricted to real analysis.

Hereafter, we present a simple two-part theoretical framework borrowed from philosophy and from mathematics education literature. Although it is not our intent to give an extensive report of either study, we next indicate the methodology used in Edwards's dissertation and in our joint abstract algebra study so that the reader may know the context from which our observations are drawn. We then list the "surprising" difficulties of the two groups of students, documenting them with examples from the studies and using the framework to provide a possible explanation for them. We conclude with what we see as the implications for undergraduate teaching, along with some specific classroom activities that the studies and our experience as teachers suggest might be of value.

2. FRAMEWORK. It is commonly noted in mathematics departments that undergraduate mathematics majors often experience difficulties when trying to write mathematical proofs in their introductory abstract algebra, real analysis, or number theory courses. Some researchers have investigated certain aspects of students' understanding or success in proof-writing [8], [16], [11]. In particular, Moore [11] notes that, while attempting to write formal proofs, students do not necessarily understand the content of relevant definitions or how to use these definitions in proof-writing. Edwards's study 
with real analysis students [5] found that some undergraduate mathematics majors, even those who would be deemed successful based upon their grades, had difficulties understanding the role that mathematical definitions play in mathematics in general. There were particular difficulties in understanding the philosophical categorization of mathematical definitions and in using definitions to perform mathematical tasks, such as proving theorems.

Definitions play a key role in mathematics, but their creation and use differ from those of "everyday language" definitions. First, we turn to philosophers and lexicographers for useful categorizations and observations about definitions. Afterward, we look to mathematics education literature for some insights on the use of mathematical definitions.

Philosopher Richard Robinson [15] distinguishes between lexical and stipulative definitions. Robinson writes "Lexical definition is that sort of word-thing definition in which we are explaining the actual way in which some actual word has been used by some actual person" [15, p. 35]. Lexicographer Sidney I. Landau [10] discusses the same category, but uses the term extracted definitions in place of lexical definitions because they are "definitions that are based on examples of actual usage, definitions extracted from a body of evidence" [10, p. 165]. Herein, we follow Landau's terminology since Edwards used "lexical" in a different way in her earlier work.

By contrast, Robinson uses stipulative definition to "mean the explicit and selfconscious setting up of the meaning-relation between some word and some object, the act of assigning an object to a name (or a name to an object)" [15, p. 59]. Its chief advantage is "the improvement of concepts or the creation of new concepts, which is the key to one of the two or three locks on the door of successful science" [15, p. 68]. Landau says such definitions "are imposed on the basis of expert advice" with the goal of "ease and accuracy of communication between those versed in the language of science" [10, p. 165].

Thus, extracted definitions report usage, while stipulated definitions create usage, indeed create concepts, by decree. Moreover, when a term is defined by stipulation, it is to be free from connotation, that is, free from all the associations the term may have acquired in its nontechnical use. We think of mathematical definitions as stipulated, whereas most "everyday language" definitions are extracted.

We turn next to the mathematics education literature to describe how students use definitions in mathematics. According to Vinner [21] and Tall [19], each mathematical concept has associated with it a concept definition and concept image. ${ }^{1}$ The concept definition can be the stipulated definition assigned to a given concept. The concept image, on the other hand, is a nonverbal representation of an individual's understanding of a concept. It includes the "visual representations, the mental pictures, the impressions and the experiences associated with the concept name" [21, p. 68]. We agree with Vinner in believing that mathematics instructors generally would imagine their students' concept image as growing out of a given concept definition, as illustrated in the simple diagram of Figure 1.

When faced with a task involving a concept, rigorous mathematics demands that concept usage follow one of the schemes charted by Vinner in Figures 2-4. The tasks we have in mind, for example, are proving theorems or identifying an object as having a certain defined structure, such as being a group. The key element is that in whatever ways the concept image enters into the processing and completion of the task, the ultimate output will be based solely on the concept definition.

\footnotetext{
${ }^{1}$ Our brief summary is based on our interpretation of Vinner [21], where the interested reader will find a more complete exposition.
} 


\section{Concept Definition}

Concept Image

Figure 1. The idealized development of a formal mathematical concept [21].

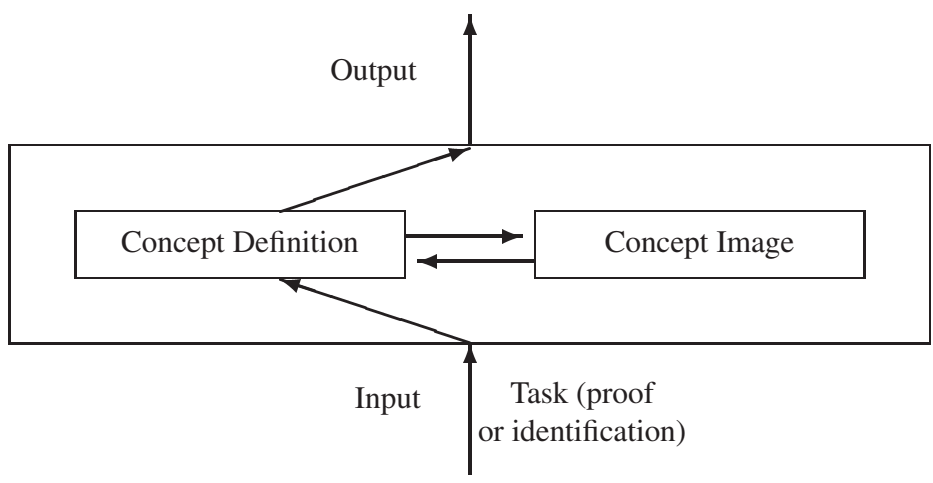

Figure 2. Interplay between definition and image [21].

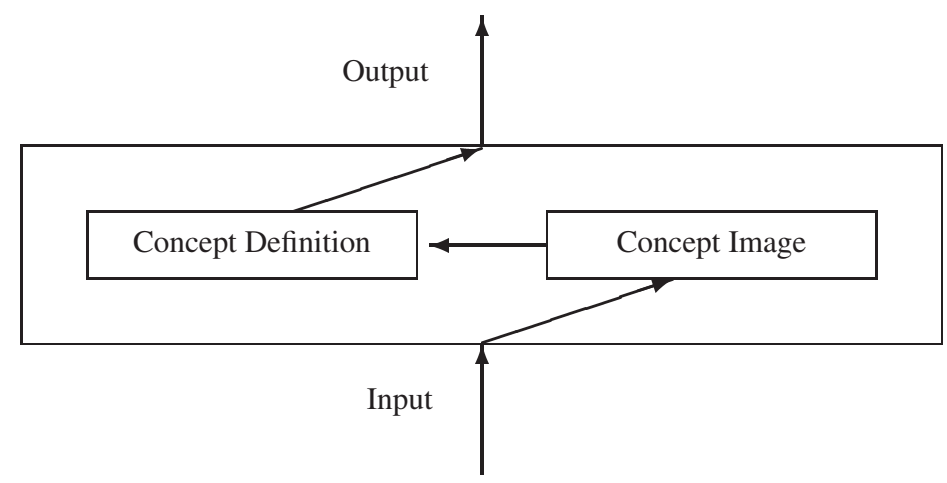

Figure 3. Deduction following intuitive thought [21].

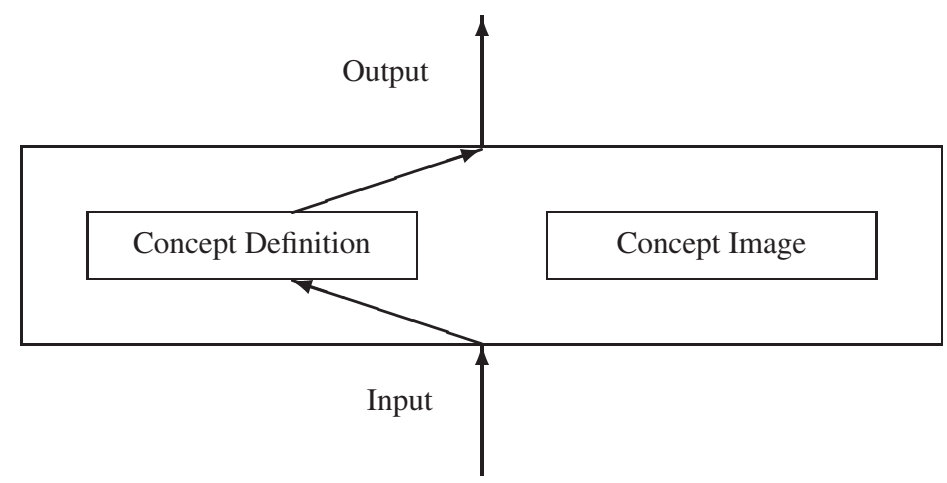

Figure 4. Purely formal deduction [21]. 
To summarize, we have introduced the categories of stipulated definitions, to which mathematical definitions belong, and extracted definitions. We have also provided cognitive models, based on concept definition and concept image, of some aspects of the processes by which definitions are used in the completion of mathematical tasks.

3. METHODOLOGY. The purpose of both studies was to look beneath student understanding of the content of mathematical definitions to discern their understandings of the role played by formal definitions in mathematics. This is somewhat tricky since it is possible that a student might apply a definition in a mathematically incorrect way for at least two reasons. It could be an incomplete or faulty understanding of the concept that it defines. On the other hand, it could be a mathematically incorrect understanding of the role of mathematical definitions in general. A further difficulty influencing the design of these studies arose from the possibility that, if asked directly, students might profess a seemingly adequate understanding of the role of formal definitions in mathematics without really understanding this role. It is not uncommon for students (or any person for that matter) to repeat something they have heard without full understanding. For instance, students may say, perhaps to please instructors, that "mathematics is necessary in all walks of life," without being able to give one meaningful example beyond the day-to-day interactions involved in commerce.

Both studies employed similar research methods. For the methodology of the real analysis study see [5] and [6]. In the algebra study, the participants were enrolled in an "Introduction to Group Theory" course taught by Ward and observed by Edwards. Data for the study were in the form of two written class assignments ${ }^{2}$ in which all fourteen students participated and interviews with eight student volunteers from the class. Each of these eight students participated in two one-hour task-based interviews (see the appendix). The first interview began with the question "What is mathematics?" By asking follow-up questions as needed, the interviewer made sure each student said something about the nature and role of definitions in mathematics. In both interviews students were asked to read a set of definitions that either had been or were to be introduced in their class, after which they were asked to work on a task related to the given definitions. We chose the definitions and tasks in an attempt to create situations in the interviews in which students might have a conflict between their concept image and the concept definitions. We also selected definitions and tasks that our experience suggested would be difficult for the students. Our particular interest was to observe whether and, if so, how the students used the definitions in overcoming their difficulties. Students had access to the written definitions at all times during the interview. The interviews were audio-taped and video-taped. Verbatim transcripts of the interviews were made and analyzed.

Analysis of the interview transcripts involved the following process. Independently, we read and reread the transcripts, studied the students' written responses, and did an initial categorization based upon an interpretation of each student's expressed view on the role of mathematical definitions and his or her mathematical behavior while working on the given tasks. We wrote brief case studies for each of the eight students and then met to compare our initial findings. We discussed our findings for each student and came to an agreement on a coding scheme to use for further study. Working individually, we then reread the transcripts using our coding scheme and met several times after this to discuss and refine our conclusions about each student. It was from comparing these results with each other and with Edwards's dissertation conclusions that the surprises emerged.

\footnotetext{
${ }^{2}$ The written exercises are not discussed in this paper.
} 


\title{
4. THE SURPRISES.
}

\section{Surprise 1: Many students do not categorize mathematical definitions the way mathematicians do.}

Mathematicians understand (and would be hard pressed to remember a time when they did not understand) that mathematical definitions are stipulated. As Robinson puts it $[15$, p. 59]:

\begin{abstract}
Above all, the mathematicians, ever since Euclid at latest, have been making their own meanings for words. 'By a denumerable series', they say, for example, 'we shall mean a series which you can put into one-to-one correspondence with the positive integers without changing its order.' This is not a historical description of what has been meant by 'denumerable' in the past or is commonly meant by it now. It is an announcement of what is going to be meant by it in the present work, or a request to the reader to take it in that sense.
\end{abstract}

Mathematics instructors may not be aware that some students do not categorize mathematical definitions among stipulated definitions. Indeed, this study came about by Ward betting Edwards that students in abstract algebra would have no choice but to treat the definitions as stipulated. To illustrate how some students fail to place mathematical definitions firmly in the stipulated category, we cite two examples from our abstract algebra study and one from Edwards's dissertation study.

Asked "What is mathematics?" in the first interview of the abstract algebra study, Andre ${ }^{3}$ brought up the notions of theorem and definition. He said, "That is basically what a theorem is, a definition.... Once [a theorem] is proven, it becomes a definition .... At some point in time, we proved that 1 plus 1 is 2 . Therefore, the definition is that always 1 plus 1 equals $2 \ldots$... At first, Andre's response seemed so strange that we were tempted to regard it as an outlier. However, by thinking in terms of the categorization of definitions, one can explain Andre's point of view. For Andre, mathematical definitions reported facts. Andre was a student for whom all properties of a mathematical object held "by definition." His definitions were extracted, though not from common usage, but rather from the body of knowledge about the concept. They were not stipulative in any sense.

Heidi, another student in the algebra study, provides another example of a student who seemed not to categorize mathematical definitions the way mathematicians do. However, she seemed to teeter on the cusp of understanding. We see her case as evidence that a student's understanding of the categorization of mathematical definitions is not necessarily clear cut. Students do not fit nicely into one group (those who understand) or the other (those who do not understand). We think of this as a transition stage similar to what Burger and Shaughnessy [4] describe as transition stages of students understanding between van Hiele levels in geometry. According to Burger and Shaughnessy, it is possible that students may exhibit different levels of understanding on different tasks and some may even oscillate between levels of understanding on the same task.

The depth of Heidi's understanding of the role of mathematical definitions was unclear to us after analyzing statements she made in her first interview. Sometimes she seemed to be exhibiting a mathematically correct view of definitions, as when she talked about a hierarchy of definitions and axioms being used to prove theorems. But a few lines later she said, "You have to make the definitions from what something actually is."

\footnotetext{
${ }^{3}$ All student names are pseudonyms.
} 
In her second interview, Heidi was presented (for the first time) with the definition for coset multiplication (see the appendix, Definition 4). Before the interview, the definition of coset had been discussed in class (appendix, Definition 2). Heidi had calculated cosets and had observed some of their properties as part of a classroom activity. Ward had proved the basic properties of cosets in lecture, and Heidi had probably done some homework on cosets. Before she could even begin dealing with the coset multiplication definition, which was what we wanted to observe, she had to calculate some cosets. Unfortunately, she could not calculate them. She worked unsuccessfully for more than twenty minutes. Edwards asked her why the definition of "coset," which was in front of her, was not helping her find the cosets. She replied, "Because I know how to do it, because I did it...." In her struggles, she repeatedly made similar statements. She did not seem to read the definition until pointedly prompted to do so by the interviewer.

Heidi's insistence on trying to remember how to compute cosets rather than to look at the definition was, perhaps, partly explained by embarrassment or a feeling that by now she ought to know the definition. However, taking into consideration the ambiguity in her descriptions of definitions in her first interview, another factor appeared to be at work. Having seen repeated instances of cosets over a period of days, she may have believed the definition should no longer be needed. Having been repeatedly shown instances of a chair, one does not need a definition in order to build or sit in a chair. Heidi seemed to believe that mathematical objects could be defined in the same way and that, if necessary, she should have been able to extract the definition from the instances.

Stephanie, a participant in Edwards's dissertation study (whose case is also discussed in [6]), seemed to believe that mathematical definitions were more like extracted definitions than like stipulated definitions. Just as a lexicographer must document a word's extracted usage before it becomes a dictionary entry, Stephanie said that, when a mathematician writes a definition, "you would have to like have your colleagues, like review it, make sure it's legitimate and there's no errors."

Andre, Heidi, and Stephanie were competent students. In fact, two of them earned high grades in their mathematics courses. Nevertheless, they categorized mathematical definitions incorrectly. All of them experienced difficulties that could be explained partly by their failure to place mathematical definitions firmly in the stipulated category.

The readers may now wish to think of their own experiences with student misuse of definitions and consider the role miscategorization may play in that misuse.

\section{Surprise 2: Many students do not use definitions the way mathematicians do, even when the students can correctly state and explain the definitions.}

The improper use of definitions is not a surprise. In an extreme (but familiar) case, definitions are not used at all! Anyone who has ever taught an advanced mathematics course will have seen students complete tasks as in Figure 5. Vinner calls that the "Intuitive Response." Most mathematics instructors would assume such a response occurs when the student does not know or does not understand the definition. Instructors generally assume that if a student can accurately state and explain a definition, then victory is at hand and such purely intuitive responses will be banished, as will other misuses of definitions. Our data, alas, suggests otherwise. That is a surprise.

For example, in Edwards's study, Stephanie was able to explain the definition of infinite decimal given in her real analysis course. ${ }^{4}$ She could use the definition to

\footnotetext{
${ }^{4}$ The definition was: Let $c_{1}, c_{2}, \ldots, c_{n}, \ldots$ be an infinite sequence of integers with $0 \leq c_{i} \leq 9$. The number $\sup \left\{0 . c_{1} c_{2} \ldots c_{n} \mid n=1,2,3, \ldots\right\}$ is denoted by $0 . c_{1} c_{2} \ldots c_{n} \ldots$ and is called an infinite decimal.
} 


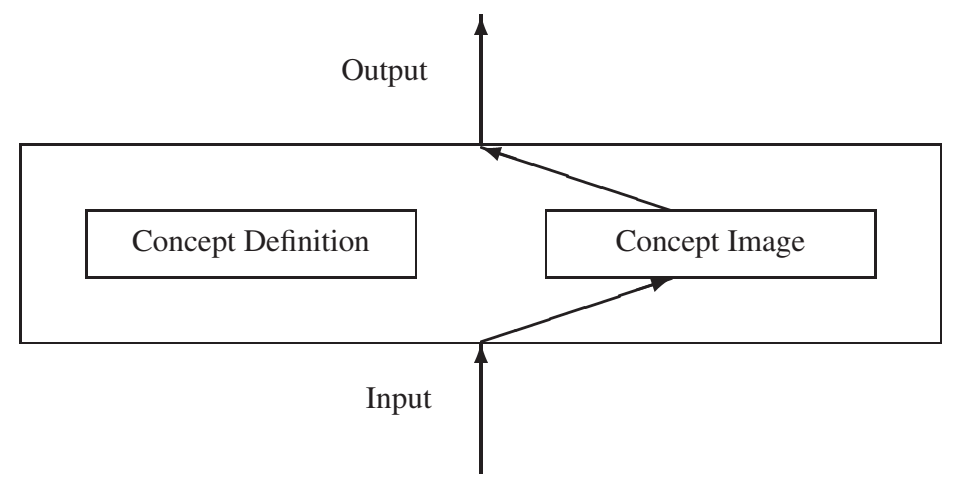

Figure 5. Intuitive response [21].

explain why $0.333 \cdots=1 / 3$ (see [6] for details). Nevertheless, she disregarded the definition when she argued that $0.999 \ldots$ was not equal to one. Instead, she used her concept image, which was based on long division. One can get $0.333 \cdots$ by dividing 1 by 3 , she said, but one does not get $0.999 \ldots$ by dividing 1 by 1 . The definition in front of her was ignored. When the concept definition conflicted with her concept image, the concept image won. Consistent with her categorization of mathematical definitions, she seemed to think the concept definition for repeating decimal had not been extracted quite correctly in the case of $0.999 \ldots$.

Similarly, in the same study, Jesse gave an acceptable definition of continuity, yet used his concept image in claiming the absolute value function is not continuous at 0 . Even though he kept saying that according to the definition the function should have been continuous, in the end, he placed more value on a memory from his introductory calculus course that something was "different" about the absolute value function.

\section{Surprise 3: Many students do not use definitions the way mathematicians do, even in the apparent absence of any other course of action.}

Vinner writes, "It seems to us that many teachers at the secondary and the collegiate levels expect a one way process for the concept formation as shown in Figure 1, namely, they expect that the concept image will be formed by means of the concept definition and will be completely controlled by it" [21, p. 71]. That was certainly what Ward thought. He had no doubt about it in the case where the definition is simply a formula, as for coset multiplication (appendix, Definition 4). He had little doubt about it in general for a first course in abstract algebra, for terms like "group" and "ring" therein defined certainly could carry no connotation on which to build a concept image independent of the definition. He attributed the difficulties of Stephanie and Jesse to entrenched, flawed concept images formed by prior experience with repeating decimals and continuity. In the absence of any prior experience, Ward expected the interviews for the algebra study to show a concept image being formed and controlled by the concept definition. There seemed to be no alternative.

We selected the definition of coset multiplication for use in our second interview because it is a known trouble maker in group theory. Some sources of the trouble have been studied in [3] and [2]. For students who might not have difficulty with the content of the definition, we ensured trouble of another kind by asking the students to consider a situation in which coset multiplication was not well-defined. Whatever the source of the trouble, our particular interest was in observing whether and, if so, how the students used definitions as tools in dealing with the trouble. 
Heidi, after finally calculating the necessary cosets, read the definition of coset multiplication and said, "Would you do it like in a FOIL form...?" FOIL, First-OuterInner-Last, refers to the distributive property for multiplying two binomials. Here it amounts to elementwise multiplication of the two cosets. (Ironically, in some cases, Heidi's scheme is actually equivalent to the given definition, a fact that is not entirely obvious.) Andre did a similar computation (under the moniker "Marvin the Martian" because the lines he drew to designate the products formed an alien-looking face).

Blake, another student in the algebra study, had a sophisticated understanding of the structure of mathematics. His description of the nature of mathematical definition was on target. In both interviews, he repeatedly sought answers in the given definitions, making comments like "It has got to be in this definition...." Nevertheless, realizing that the operands in coset multiplication are sets, he wondered for some time if the definition of coset multiplication was only a "weird way" to indicate "simply listing them [the coset elements] all out," by which he meant forming the union of the two cosets.

For Heidi and Andre, the coincidental fact that each coset in the problem we presented contained two elements led them to write expressions such as $\{V, H\}\{D, F\}$. Presumably, that cued the FOIL response because of the visual similarity with $(V+H)(D+F)$. For Blake, the fact that two sets were being operated together brought to mind another set operation, union. He then tried to imagine how the given formula might describe union. For all three students, the concept image for coset multiplication was not formed as envisioned in Figure 1, even though we conjectured there would be no alternative. Instead, we saw the appearance of an inappropriate, preexisting, concept image, namely, either FOIL or union. The inappropriate concept image competed with or may even have replaced the concept definition as the controlling force in the formation of the concept image (see Figure 6). Upon reading an unfamiliar mathematical definition, an experienced mathematician is more likely to form a concept image (at least a partial concept image) based solely on the concept definition and then afterward to explore its relationship to existing images. Heidi, Andre, and Blake did not use the concept definition in the way an experienced mathematician would use it.

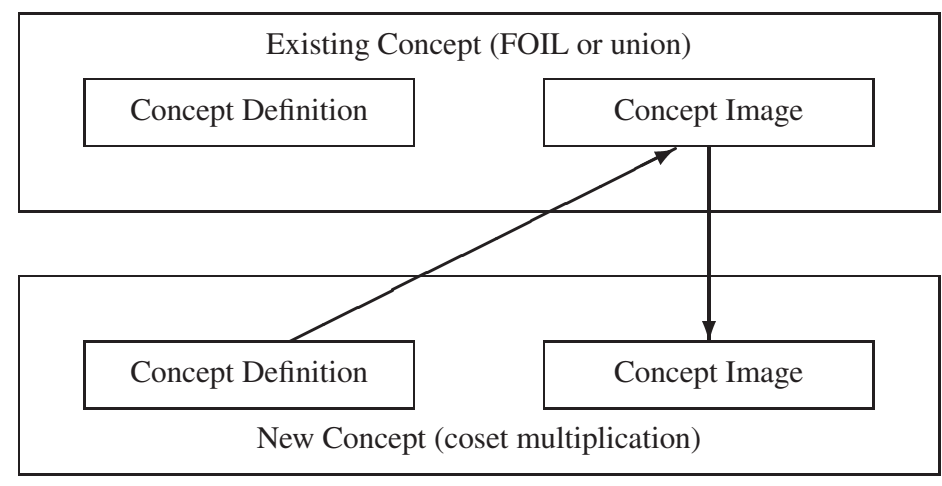

Figure 6. Inappropriate image influencing image formation.

5. IMPLICATIONS FOR TEACHING. In this section we give some implications for teaching inspired by these studies. We also suggest some sample classroom activities. Investigating the effectiveness of these suggestions could be the basis for future research. 
Implication 1. The special nature of mathematical definitions should be treated as a concept in its own right, one that should be understood at some level by all college mathematics students.

This is especially important in a student's first proof-intensive course, which we refer to generically as an "introduction to proof" course. Certainly a case can be made for insisting that students know the exact content of the definitions they use, but this is not sufficient. It is very clear from our analysis of the data in these studies that students also need to understand the specific role definitions play in mathematics. Thus in such courses the nature of mathematical definitions needs to be addressed more directly and more often.

In particular, introduction to proof courses certainly should include a unit on the categorization and use of mathematical definitions. The introductory material in this article should be sufficient for describing the categorization of definitions, although reading chapter 4 of Robinson [15] for additional background would be very enlightening for the instructor. Chapter 3 in Solow [17] and chapter 3 (especially pages 82-83) in Exner [7] illustrate methods for teaching the use of mathematical definitions.

It may be informative for students (and instructors) to search the dictionary for words that have both extracted and stipulated definitions. For instance, The American Heritage Dictionary [1] lists both extracted and stipulative definitions for the word "radical," namely, "excellent; wonderful" and "the root of a quantity as indicated by the radical sign," respectively. This is an especially good example because it shows how the use of words over time can evolve new extracted definitions. The definition "excellent; wonderful" is not found in dictionaries printed thirty years ago. Mathematical definitions are not extracted from popular usage in the way "excellent" has become a definition for "radical." Though mathematical definitions can vary over time and context, mathematicians are careful to stipulate which definitions are to be used in their own work.

There are other available activities that focus on the nature of mathematical definitions. We give a few recommendations here.

A short version of the junior high activity in [12] played out very well for Ward, even in an upper-division axiomatic geometry course. It quickly illustrates why mathematical definitions are and must be stipulated with such precision. The activity went as follows.

Question from Ward: What is a quadrilateral?

Response from the class: A four-sided figure.

Ward drew a four-sided figure with curved sides.

Response: Oops, a four-sided figure having straight line segments as sides.

Ward drew an open four-sided figure ...

The activity continued in this fashion until an acceptable definition was given. (Reference [12] gives further details and follow-up activities.)

Even more to the point are activities that engage students in actually formulating definitions in the same way that practicing mathematicians do. For example, Edwards uses David Henderson's geometry text [9] in a course for mathematics majors who intend to teach either high school or community college mathematics. Early in the course students have a definition of "triangle" that is useful for a while on the Euclidean plane, on the sphere, and on the hyperbolic plane, even though triangles on the sphere can sometimes look a good deal different than the triangle on the plane that we have seen since elementary school. However, students eventually find that the SideAngle-Side Theorem (SAS) is not true for all triangles on the sphere. At this point 
they must do what all mathematicians do-look for the special case or cases for which the theorem is valid. This leads to a special definition of a "small triangle," one for which SAS is true on the sphere.

Implication 2. Instructors can better understand the struggles undergraduate students face in proof-intensive courses by thinking in terms of the required shift to the concept definition-based logical reasoning illustrated in Figures $2-4$. The students very likely have thirteen years of conditioning in courses where Vinner's Intuitive Response (Figure 5) is closer to the norm. This transition can be difficult, not to mention puzzling, for many students.

A brief presentation (like the one herein, for example) on concept definition and concept image might provide students with a framework in which to think about what is happening in their classes. In fact, Vinner's discussion on concept definition and concept image [21, pp. 68-73] is probably accessible enough to be of value to an undergraduate. That presentation might be done in conjunction with a reading on the centrality of rigor and proof in mathematics such as is found in Stewart ("What Mathematics Is About" [18, chap. 3]) in order to explain why the shift to increased rigor is necessary.

Ward used the material from Vinner in two recent courses, an upper-division course in axiomatic geometry and an introduction to proof course. In geometry, he spent about twenty minutes on the first day of class discussing concept image versus concept definition and showing Figures 1-5. Most of the students immediately adopted Vinner's terminology. When asking about a step in a proof, many would say "My concept image is ... [usually referring to a diagram]. How do I make that rigorous?" When an unjustified claim was made in a proof (for example, " $A B C D$ is a quadrilateral"), Ward would ask: "Is that based on concept image (the diagram) or concept definition $(A, B, C$, and $D$ are distinct coplanar points, no three of which are collinear, and any pair of the segments $A B, B C, C D$, and $D A$ either have no points in common or have only an endpoint in common)?" The students all seemed to see the point of such questions.

In the introduction to proof course, Ward introduced the concept image/concept definition terminology and Vinner's figures late in the course after several structural proof techniques (in the style of Velleman [20], Solow [17], or Exner [7, chap. 3]) had been introduced. He referred to various proofs done earlier in the course and showed how the diagrams modeled the process used in class to produce the proofs. For example, in proofs about sets, Venn diagrams provided a useful part of a concept image in some cases. Figure 2 or Figure 3 modeled the process by which those proofs were constructed. For proving injectivity and surjectivity of functions, Ward had stressed how a proof strategy could be outlined by simply looking at the quantifiers in the definitions. He then related that to Figure 4. For the students who continued to write heuristic, arm-waving pseudo-proofs, Ward pointed to Figure 5.

Implication 3. The results of this research should be considered in the mathematical preparation of future teachers.

One might ask if it is sufficient to wait until students are enrolled in post-calculus mathematics courses to begin talking about the special nature of mathematical definitions? In Edwards's dissertation study with students in a beginning real analysis course, Jesse provided some evidence against waiting. Especially in Jesse's early interviews, he displayed a belief (and even acted accordingly) that the words of a definition were secondary to one's understanding of the related concept. To use the terminology of Vinner, if one's concept image conflicted with the related concept definition, Jesse 
believed the concept image should prevail. Jesse repeatedly attributed his understanding of the nature of mathematical definitions to what he had learned in his high school mathematics courses. As he explained in the second interview, his AP calculus instructor had talked early in the course about the notion of formal mathematical definitions. But Jesse said, "After about the first day in calculus, we didn't care about this [formal definition] if you had the concept right, not really the definition, that was all that really mattered." And again in the third interview he said, "[the calculus teacher] would even say, now this [the formal definition] may mean a lot of jargon, but this is what it really means." We can probably understand the motives of the instructor. It is important for students to have deep conceptual understandings. Jesse's instructor probably did not want his students to be hindered by what often seems to be the heavily symbolic and terse language of formal definitions, but he was also assuming (or hoping?) that his students would not leave his course with mathematically incorrect understandings of the concepts in calculus.

The goal of helping students develop deep conceptual understandings does not have to conflict with helping students see the special role played by definition in mathematics. Indeed, The National Council of Teachers of Mathematics Standards recommend that "Teachers can help students see that some words that are used in everyday language, such as similar, factor, area, or function are used in mathematics with different or more-precise meanings. This observation is the foundation for understanding the concept of mathematical definition" $[13$, p. 63]. In other words, the standards advise $\mathrm{K}-12$ mathematics teachers to inform students of the need to beware of connotations a word may carry because of its nontechnical use and also of the need to distinguish informal concept images from precise concept definitions.

Furthermore, it is worth noting a common method of creating definitions that seems related to extracted definitions. One defines ostensively [15, pp. 117-126], [10, pp. 167-168] when one indicates something, with a gesture perhaps, and says "this is a..." With enough repetition, one expects the listener to pick up the meaning. Recall that Heidi seemed to be under the impression that cosets could be defined ostensively.

Since many mathematics majors will eventually teach mathematics at some level, they should be made aware of the dangers of defining ostensively. Prevost reports on high school sophomores who were repeating a geometry course during the summer, having failed it the previous year. He writes, "Almost all the students could parrot the definitions they had learned during the previous year. Their 'working definition,' however, was 'looks like.' ... As teachers, we are guilty of reinforcing this 'looks like' definition. The figures we draw are stereotypical and oriented with one side parallel to the lower edge of the paper or chalkboard" [14, p. 412]. Thus, for instance, a square whose sides are not parallel to the sides of the paper was identified by the students as "a diamond, perhaps, but not a square" [14, p. 411]. In the terminology of this paper, in spite of knowing the stipulated definitions of geometric figures, those students worked as though the figures had been defined ostensively by repeated reference to stereotypical instances of figures in certain orientations. They remained at van Hiele's "recognition" level of geometric sophistication rather than advancing to the "analysis" level. (See [4] for a discussion of the van Hiele levels.) Not surprisingly, Prevost relates this failure at accurate identification to failure in the course.

We certainly are not calling for the use in $\mathrm{K}-14$ classrooms of the so-called definition-theorem-proof means of discourse that is prevalent and valuable among working mathematicians. What we are saying is that teaching for conceptual understanding is valuable but should not be done at the expense of students' building a good understanding of the nature and use of mathematical definitions. Even before students take proof-intensive courses and even before college, instructors should attempt to 
plant the seeds of a more rigorous approach to the use of definitions. Thus, it is particularly important that undergraduate mathematics students who plan to teach should have experiences in their college courses that help them build robust understandings of the role and use of mathematical definitions.

6. CONCLUSION. We have used terms from the literature on definitions to categorize definitions as extracted (everyday language) or stipulated (mathematical). Our research has shown that undergraduate mathematics majors may not fully understand this distinction, and that this failure to understand affects their understanding of the concepts themselves. The tendency of some of the real analysis students in Edwards's original study to rely on their concept images instead of the related concept definitions, when the two were in conflict, could be partially explained by the fact that some of the definitions used in that study were for concepts that were very familiar to the students from their previous mathematics courses (continuity and infinite decimal, for example). This was not the case in the abstract algebra study, yet some of the algebra students displayed a similar preference for concept image over concept definition.

We have concluded, therefore, that the special nature of mathematical definitions should be addressed more directly in mathematics courses at all levels, but especially in the first proof-intensive course. Students should have experiences that focus on the use of mathematical definitions and experiences in the process of defining. Although it may be true that many students eventually "figure out" the role of mathematical definitions, it seems that it would be preferable not to leave this important facet of the nature of mathematics to chance.

\section{APPENDIX.}

\section{Abstract Algebra Interview 1}

The first interview began with Edwards asking "What is mathematics?" By asking follow-up questions as needed, Edwards made sure each student said something about the nature and role of definitions in mathematics.

After that introductory discussion, each student was handed the following definition. Binary operations were being studied in class, but the definition of group had not yet been presented.

Definition 1. Let $G$ be a nonempty set together with a binary operation $\star$ that assigns to each ordered pair $(a, b)$ of elements of $G$ an element of $G$ denoted by $a \star b$. We say $G$ is a group under $\star$ if the following three properties are satisfied.

1. The operation is associative; that is, $(a \star b) \star c=a \star(b \star c)$ for all $a, b, c$ in $G$.

2. There is an element $e$ (called the identity) in $G$, such that $a \star e=e \star a=a$ for all $a$ in $G$.

3. For each element a in $G$, there is an element $b$ in $G$ (called the inverse of $a$ ) such that $a \star b=b \star a=e$.

After reading the definition, each student was given these three tasks to complete.

Set: $\mathbf{R}$, the set of real numbers

Binary Operation: Ordinary subtraction, -

Is $\mathbf{R}$ a group under subtraction? 
Set: $\mathbf{R}$, the set of real numbers

Binary Operation: The operation $\oplus$ where $a \oplus b=a+b+3$

Is $\mathbf{R}$ a group under $\oplus$ ?

Set: $\mathbf{R}$, the set of real numbers

Binary Operation: Ordinary multiplication

Is $\mathbf{R}$ a group under multiplication?

\section{Abstract Algebra Interview 2}

In addition to Definition 1, the following definitions were given in the second interview. The last two were new to the students.

Definition 2. Suppose $K$ is a subgroup of a group $G$ and $a$ is an element of $G$. The set $\{$ ay $: y \in K\}$ is called a left coset of $K$ in $G$. It is denoted by $a K$.

Definition 3. Suppose $K$ is a subgroup of a group $G$. We define $G / K$ to be the set of all left cosets of $K$ in $G$. In other words, $G / K=\{a K: a \in G\}$.

Definition 4. Suppose $K$ is a subgroup of a group $G$. For left cosets $b K$ and $c K$ of $K$ in $G$, we define $(b K)(c K)=b c K$.

Each student was given the following task to complete based on the definitions. The symmetry group of the square is denoted by $D_{4}$, and $F$ denotes a reflection across one of the diagonals.

Consider the subgroup $\langle F\rangle$ of the group $D_{4}$ under the operation of composition.

1. Find $D_{4} /\langle F\rangle$.

2. Is $D_{4} /\langle F\rangle$ a group where $(b\langle F\rangle)(c\langle F\rangle)$ is defined by Definition 4 ?

ACKNOWLEDGMENT. The authors gratefully acknowledge support from the National Science Foundation and the Oregon Collaborative for Excellence in the Preparation of Teachers (DUE-9653250).

\section{REFERENCES}

1. The American Heritage Dictionary of the English Language, 4th ed., Houghton Mifflin, Boston, 2000.

2. M. Asiala, E. Dubinsky, D. Mathews, S. Morics, and A. Oktac, Student understanding of cosets, normality and quotient groups, J. Math. Behavior 16 (1997) 241-309.

3. A. Brown, D. DeVries, E. Dubinsky, and K. Thomas, Learning binary operations, groups, and subgroups, J. Math. Behavior 16 (1997) 187-289.

4. W. Burger and J. M. Shaughnessy, Characterizing the van Hiele levels of development in geometry, $J$. Research in Math. Education 16 (1986) 31-48.

5. B. Edwards, Undergraduate mathematics majors' understanding and use of formal definitions in real analysis, unpublished doctoral dissertation, Pennsylvania State University, 1997.

6. - An undergraduate student's understanding and use of mathematical definitions in real analysis, in Proceedings of the Nineteenth Annual Meeting of the North American Chapter of the International Group for the Psychology of Mathematics Education, vol. 1, ERIC Clearinghouse for Science, Mathematics and Environmental Education, Columbus, OH, 1997, pp. 17-22.

7. G. R. Exner, An Accompaniment to Higher Mathematics, Springer-Verlag, New York, 1996.

8. G. Harel and L. Sowder, Students' proof schemes: Results from exploratory studies, in Issues in Mathematics Education Vol. 7: Research in Collegiate Mathematics Education. III, A. H. Schoenfeld et al., eds., American Mathematical Society, Providence, 1998, pp. 234-383. 
9. D. W. Henderson, Experiencing Geometry in Euclidean, Spherical and Hyperbolic Spaces, 2nd ed., Prentice Hall, Upper Saddle River, NJ, 2001.

10. S. I. Landau, Dictionaries: The Art and Craft of Lexicography, 2nd ed., Cambridge University Press, Cambridge, 2001.

11. R. C. Moore, Making the transition to formal proof, Educational Studies in Math. 27 (1994) 249-266.

12. L. Pereira-Mendoza, What is a quadrilateral?, Math. Teacher 86 (1993) 774-776.

13. Principles and Standards for School Mathematics, The National Council of Teachers of Mathematics, Reston, VA, 2000.

14. F. J. Prevost, Geometry in the junior high school, Math. Teacher 79 (1985) 411-417.

15. R. Robinson, Definition, Oxford University Press, London, 1954; reprinted by D. R. Hillman \& Sons, Frome, U.K., 1962.

16. A. Selden and J. Selden, Validations of proofs considered as texts: Can undergraduates tell whether an argument proves a theorem? J. Research in Math. Education 34 (2003) 4-36.

17. D. Solow, How To Read and Do Proofs: An Introduction to Mathematical Thought Processes, 3rd ed., John Wiley \& Sons, New York, 2002.

18. I. Stewart, Nature's Numbers: Discovering Order and Pattern in the Universe, Weidenfeld \& Nicholson, London, 1995.

19. D. Tall, The transition to advanced mathematical thinking: Functions, limits, infinity and proof, in NCTM Handbook of Research on Mathematics Teaching and Learning, D. A. Grouws, ed., Macmillan, New York, 1992, pp. 495-511.

20. D. J. Velleman, How To Prove It: A Structured Approach, Cambridge University Press, Cambridge, 1994.

21. S. Vinner, The Role of Definitions in the Teaching and Learning of Mathematics, in Advanced Mathematical Thinking, D. Tall, ed., Kluwer, Dordrecht, 1991, pp. 65-81.

BARBARA S. EDWARDS received her Ph.D. in mathematics education from Pennsylvania State University in 1997 (thus beginning her third career). Her research interests are in advanced mathematical thinking and the teaching and learning of undergraduate mathematics. She is an associate professor in the Mathematics Department at Oregon State University.

Oregon State University, Corvallis, OR 97331

edwards@math.orst.edu

MICHAEL B. WARD was an undergraduate at Utah State University and received a Ph.D. from the University of Utah in 1979. He worked at Bucknell University until 1997, when he moved to Western Oregon University, thereby fullfilling a twelve-year family dream of living in the offbeat state of Oregon. Besides trying to prove the occasional theorem in group theory, he enjoys reading, walking, and spending time with his wife Elizabeth and his children Hans, Liesl, and Maren.

Department of Mathematics, Western Oregon University, Monmouth, OR 97361

wardm@wou.edu 